TM-1607

\title{
Calculation of Integrated Luminosity for Beams Stored in the TEVATRON Collider
}

\author{
D. A. Finley \\ Fermi National Accelerator Laboratory \\ P.O.Box 500, Batavia, Illinois
}

March 20, 1989

Presented at the 1989 IEEE Particle Accelerator Conference, Chicago, Iflinois, March 20-23, 1989.

둘 Operated by Universities Research Association, Inc., under contract with the United States Department of Energy 
Calculation of Integrated Luninosity for Beans

Stored in the Tetatron Collider

D.A. Finley

* Fermi National Accelerator Laboratory

Batayia, Il $\mathbf{6 0 5 1 0}$

\section{Introduction}

A model for calculating the integrated luminosity of beams stored in the Tevatron collider will be presented. The wodel determines the instantaneous luminosity by calculating the overlap integral of bunched beans passing through the interaction region. The calculation accounts for the variation in beam sise due to the beta functions and also for effects due to finite longitudinal amittance and non-zero dispersion in the interaction region. The integrated luminosity is calculated for the beams as they evolve due to processes including collisions and intrabeam scattering. The model has been applied to both the extant and upgraded Tevatron collider, but is not limited to them.

The original notiration for developing the computer model was to determine the reduction in luminosity due to beams with non-zero longitudinal emittances. There are two effects: 1) The transverse beaw size is increased where the dispersion is nonzero; 2) The finite length of the beam bunch combined with an increasing $\rho$ function results in sn incressed transverse beam size at the ends of the bunch. The derivation of a sufficiently useful asalytic expression for the luminosity proved to be intractable. Instead, a numerical integration computer program was developed to calculate the luminosity in the presence of a finite longitudinal emittance. The program was then expanded into a model wich allows the luminosity to vary due to changes in emittances and reduction in bunch intensities. At that point, it was not difficult to calculate the integrated luminosity.

\section{huminosity}

\section{Instantaneous Luminosity}

A general expression for instantaneous luminosity has been given by $\mathbf{Y}$. Honth [M] :

$$
\text { c }\left[\left|\rho_{1}-\rho_{2}\right|^{2}-\left|\rho_{1} \times \rho_{2}\right|^{2}\right]^{1 / 2} \int \rho_{1} \rho_{2} \mathrm{dV},
$$

in which $\rho$ is the rolume particle density, $p c$ is the particle velocity, $c$ is the speed of light and $d V$ is $d x d y d z$. For the Tevatron it is reasonable to assume $\beta_{1}=-\beta_{2}$ which gives:

$$
2 \beta c \int \rho_{1} \rho_{2} \mathrm{dV} \text {. }
$$

The particle density can be written as [MM] :

$$
P_{i}(x, y, z, t)=N_{i} S_{i}(x, y, z) T_{i}\left(z-\delta_{i}\right) .
$$

$N_{i}$ is the total number of particles in bunch $i . S_{i}$ is the satial density function. $T_{i}$ is a dimensionless function which accounts for the displacement of the bunch from the origin. $\delta_{i}$ is given by $\pm \rho c\left(t-t_{i}\right)$, where $t_{i}$ is the time when the center of bunch $i$ reaches $s=0$. The \pm sign ambiguity is resolved by

* Operated by Universities Researcb Association, Inc. under contract with the U.S. Dept. of Energy. choosing the protons to move in the +2 direction. The origin of time is chosen to be the instant at which the center of the proton bunch crosses $z=0$.

Bunch Luminosity

The bunch luminosity is the integrated luminosity obtained from the passage of one bunch through the other [MM]. This represents integrals over space as well as time. For the Tevatron, a realistic time interval for the bunch integration is $<20 \mathrm{nsec}$.

$$
\mathrm{L}_{\text {bunch }}=2 \rho \mathrm{c} \mathrm{N} \mathrm{N}_{2} \int \mathrm{dt} \mathrm{T}_{1} \mathrm{~T}_{2} \int \mathrm{dV} \mathrm{s}_{1} \mathrm{~s}_{2} \text {. }
$$

Luminosity per Turn

If there are $B$ identical bunches in each beam around the accelerator circumference, and $f$ is the revolution frequency, the luminosity per tura can be mitten as

$$
L(t)=B L_{\text {bunch }} f \text {. }
$$

For the Tevatron, $f=47713 \mathrm{~Hz}$. The model assumes that there are no changes in the beam parameters on the time scale of one turn (about $21 \mu \mathrm{sec}$ ). $L(t)$ has units of instantaneous luminosity.

\section{Integrated Luminosity}

If $L(t)$ is the luminosity per turn at a time $t$ and $T$ is the store duration, then the integrated luminosity in the store is given by

$$
\int_{0}^{T} L(t) d t .
$$

As an example, suppose $L(t)$ had a time dependence characterized by an exponential decay witb a constant lifetime $\tau$. In this case the integrated luminosity can be derived analytically:

$$
\begin{gathered}
\text { for } L(t)=L_{0} e^{-t / \tau}, \\
\int L d t=L_{0} \tau\left[1-e^{-\tau / \tau}\right] .
\end{gathered}
$$

$A$ numerical example is given by $L_{0}=1 \times 1030 \mathrm{~cm}-2$ $\sec ^{-1}, \tau=27$ hours and $T=27$ hours (along with $10^{24}$ barn $=1 \mathrm{~cm}^{2}$ ) which yield $f$ ldt $=60$ inverse nanobarns or $60 / \mathrm{nb}$.

\section{Measured Luminosity}

However, a simple exponentixl decay does not adequately describe the behavior of the luminosity in the Tevatron as Figure 1 clearly demonstrstes. Here, on this semilog plot, the CDF sonitor of the luminosity does not have single slope in time. Indeed, the luminosity lifetime increases with time. (The curves will be discussed in a later section.)

\section{Emittances and Beam Sizes}

The spatial density function $s$ depends on the beam size in all three dimensions. At locations in the lattice where the dispersion is sero, the $95 \%$ 
normalized transverse emittance $\epsilon$ is defined to be related to the rms bean size $\left(\sigma_{0}\right)$ by:

$$
\epsilon=\frac{6 \mathrm{p} / \mathrm{m}}{f_{\mathrm{L}}} \sigma_{0}^{2} \pi .
$$

where $p$ is the beam momentum and $m$ is the beam particle rest rass. The lattice amplitude function f is given by:

$$
\rho_{L}=\rho_{0}-2 a_{0}=+\frac{1+a_{0}^{2}}{\rho_{0}} \varepsilon^{2}
$$

Table 1 lists the ralues of $\beta_{0}$ and $a_{0}$ for various Tevatron lattices at the center of the straight section in which CDF is located.

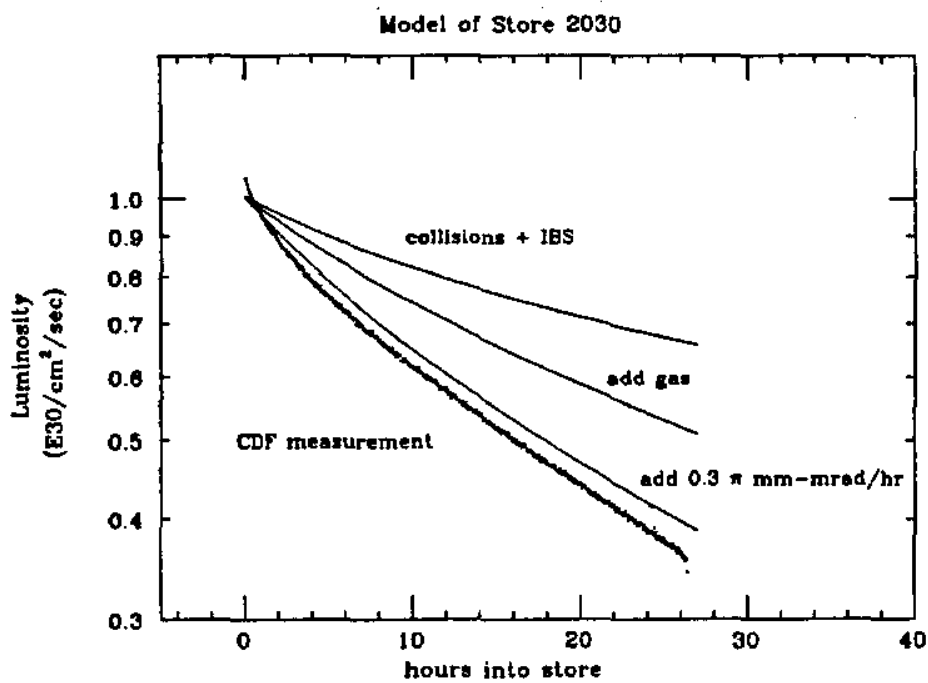

Figure 1. Time dependence of luminosity. The solid lines represent results of the model starting at $t=0$.

Table 1. Lattice Parameters.

\section{$\frac{1989 \cdot \mathrm{R} \text {. } \mathrm{n}}{\text { Injection }}$}

Pox (m) 72.3

0.427

ax $\quad-.468$

a oy $\quad 0.467$

n० (a) $\quad 2.41$

Tt $\quad 18.74$

tune 18.4

Energy $150 \mathrm{GeV}$

RP

0.163

$-.056$

0.194

$-.146$

17.88

19.4 $\eta$ \% 0.021

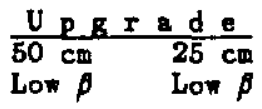

$900 \mathrm{GeV}$

$1.1 \mathrm{WV}$
0.495

0.500

0.001

0.241

0.248

0.022

0.108

$\begin{array}{ll}0.015 & 0.054 \\ 0.084 & 0.178\end{array}$

$18.60 \quad 18.60$

20.6

20.6

$1 \mathrm{TeV} \quad 1 \mathrm{TeV}$

$1.1 \mathrm{WV} 1.1 \mathrm{VV}$ function

Frow Table 1 one can see that the dispersion

$$
\eta=\eta_{0}+\eta_{0}^{\prime} z .
$$

is not sero through the interaction region. In this case, the nomentum spread due to a finite longitudinal exittance increases the transverse bean size. The model assumes the resulting rms beam size $(\sigma)$ is:

$$
\sigma^{2}=\sigma_{o}^{2}+\left(\eta \sigma_{p}\right)^{2}
$$

where $\sigma_{0}$ is the rms beam size due to transverse emittance alone and $\sigma_{p}$ is the rws momentum spread.

\section{Evolution of Luminosity}

\section{Bunch Intensities}

The bunch intensities are reduced using two effects. The first is due to proton antiproton collisions at $1.8 \mathrm{TeV}$ (which are the whole point of the collider). The loss rate is proportional to the luminosity and the cross section:

$$
\mathrm{dN} / \mathrm{dt}=\sigma_{\text {in }} \mathrm{L} .
$$

The model assumes $\sigma_{\text {in }}=44$ mbarn. The other modelled source of intensity loss is due to nuclear scattering off the gas solecules in the bean tube [MOR]. The gas is assumed to be represented by the following:

$\begin{array}{llll}\text { Warm sections } & 1 \times 10^{-8} \text { torr } & 60 \% \mathrm{~B}_{2} & 40 \% \mathrm{CO} \\ \text { Cold sections } & 5 \times 10^{-11} \text { torr } & 75 \% \mathrm{~B}_{2} & 25 \% \mathrm{He}\end{array}$

This gas composition yields a bunch intensity lifetime of 260 hours which is consistent with observations on very long stores.

\section{Bunch Emittances}

The trangverse emittances are ovolved using tbree effects: intrabeam scattering [AP], multiple Coulomb scattering of gas molecules within the beam tube [MOR], and an arbitrary source of emittance growth. The gas gives an emittance growth time of 212 hours. The longitudinal emittance evolves with intrabesm scattering only. The motions in the two transverse planes are assumed to be fully coupled.

The particular formulation of intrabeam scattering used in the model has been published by $I$. Evans [LE] and his full formulation is used in the model. However, sometimes it is convenient to have a quick estimate of the emittance growth rates due to intrabeam scattering. The following empirical expressions are adequate for such estimates if one has a Tevatron bunch of 6E10 intensity t $1 \mathrm{TeV}$ with 1 VV/tura RF voltage.

$$
\frac{d \epsilon}{d t}=C \epsilon_{x}^{-1.24} \epsilon_{p}^{-0.68}
$$

The constant $C$ depends on which emittance one is evaluating:

$$
\text { for } \epsilon=\epsilon_{x}, C=36.4 \pi \mathrm{mm}-\mathrm{mrad} / \text { hour }
$$
and for $\epsilon=\epsilon_{p}, C=9.70 \mathrm{eV}-\mathrm{sec} /$ hour.

$\epsilon_{X}$ is the horizontal $95 \%$ normalized transverse emittance and $\epsilon_{p}$ is the longitudinal emittance. The gronth rate scales with bunch intensity. Full transverse coupling causes equal sharing of the horizontal emittance growth nith the rertical; the sum of the tro emittance growths is still given by the above expression.

\section{Comparison to Present Tevatron}

Table 2 gives the observed values of intensities and emittances for a store from the present run. These are used in the model to predict the evolution of the luminosity and the curves in Figure 1 represent 
the predictions of the model as the various luminosity degradation mechanisms are added. At $t=0$ the value of the luminosity calculated by the overlap integral model agrees to $5 \%$ with the value measured by CDF. However, starting at $t=0$ hours, the collisions, intrabeam scattering (IBS) and the interactions with the gas are not sufficient to describe the time behavior of the luninosity. It is necessary to postulate enother ad hoc source of transverse emittance growth. Possible sources of this additional growth are discussed elsewhere [DH] .

Table 2. Parameters for Store 2030 Yarch 9, 1889

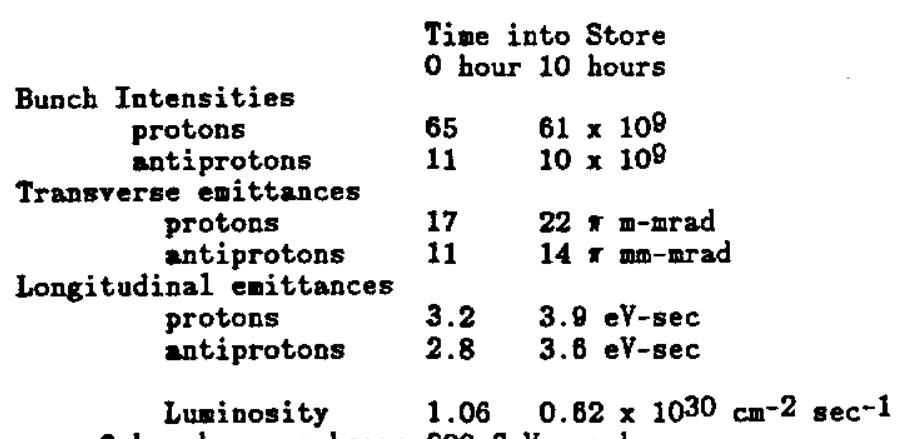

6 bunches per beam; $800 \mathrm{GeV}$ per beam

Pigure 2 shows the results of restarting the model at $t=10$ hours into the store. This gives slightly better agreement in the initial luminosity, and the luminosity behavior predicted by the model is not qualitatively different frow starting it at $t=0$.

For the first 10 hours of this store, the model predicts fLdt to be $33 / \mathrm{nb}$ from collisions and intrabeam scattering alone. Including gas scattering reduces it to $31 / \mathrm{nb}$. The ad hoc transverse emittance growth rate of $0.3 \pi \mathrm{m}-\mathrm{mrad} / \mathrm{hr}$ reduces it to $2 \theta / \mathrm{nb}$, which is very close to the measured value of $28 / \mathrm{nb}$. Restarting the podel at $t=10$ hours yields 35,32 and $30 / n b$ which again compares closely to the measured value which happens to be $28 / \mathrm{nb}$ again.

\section{Application to Collider Upgrade}

Table 3 gives beam parameters for a possible version of the collider upgrade. The upgrade lattice incorporating two low inserts of $25 \mathrm{~cm} \rho^{*}$ each is used. Table 4 shows the integrated luminosity for the various causes of luminosity degradation considered in the model. The store length is taken to be 10 hours.

\section{Conclusions}

In the present Tevatron, the integrated luminosity is determined by more than collisions losses, intrabeas scattering and gas scattering. Postulating an additional source of transverse emittance growth of about $0.3 * \mathrm{~mm}-\mathrm{mrad} /$ hour brings the model closer to the observations.

In the upgraded collider, the integrated luminosity per day could approach $1 / \mathrm{pb}$. This may be compared to the $6.6 / \mathrm{pb}$ delivered so far in the present run.

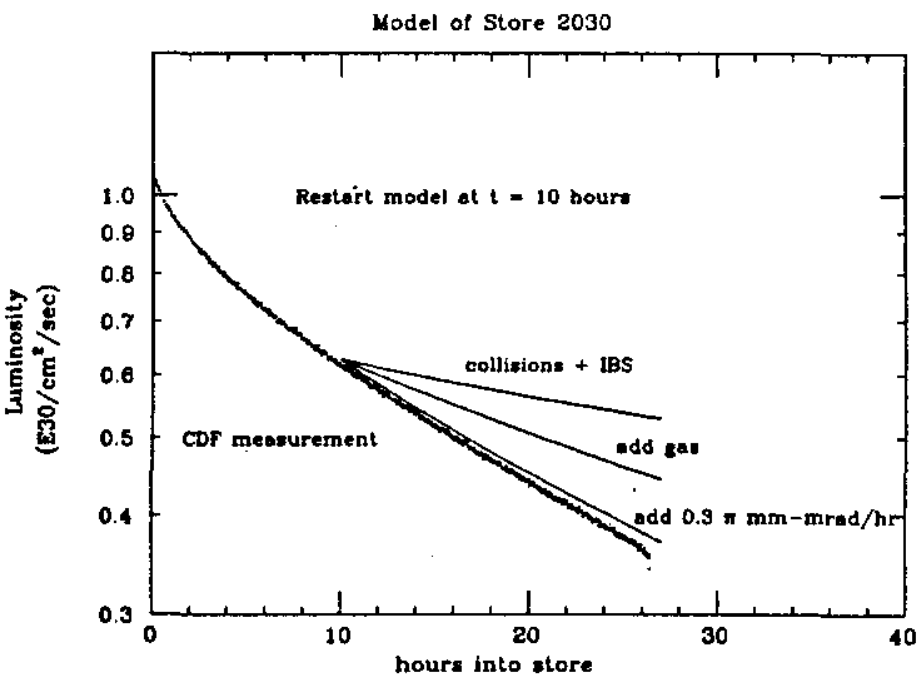

Figure 2. Same store as Figure 1 but the solid lines represent results of the model starting at $t=10$ hours.

Table 3. Upgrade Example Parameters

\begin{tabular}{|c|c|c|}
\hline & protons & antiproto \\
\hline $\begin{array}{l}\text { Intensities } \\
\text { Emittances }\end{array}$ & 60 & $50 x$ \\
\hline $\begin{array}{l}\text { transverse } \\
\text { longitudinal }\end{array}$ & $\begin{array}{l}12 \\
3\end{array}$ & $\begin{array}{l}12 \pi \text { m-mrad } \\
3 \text { eV-sec }\end{array}$ \\
\hline
\end{tabular}

22 bunches per beaw; $1 \mathrm{TeV}$ per beam $3 \times 1031 \cdot \mathrm{cm}^{-2} \mathrm{sec}^{-1}$ Initial luminosity

Table 4. JLdt for Upgrade Example

$\begin{array}{rr}\text { collisions and intrabeam scattering } & 0.87 / \mathrm{pb} \\ \text { add gas scattering } & 0.83 / \mathrm{pb} \\ \text { add } 0.30 \times \text { min-mad/hour } & 0.77 / \mathrm{pb}\end{array}$

\section{References}

[AP] A. Piwinski, Proceedings of the 9th Internationa] Conference on High-Energy Accelerators (Stanford, 1074), p. 405 .

[DH] D. A. Herrup et al., Luminosity Lifetime in the Tevatron Collider, this conference.

[LE] L. Evans, Physics of High-Energy Particle Accelerators (BNL/SUNY Summer School, 1883), AIP Conference Proceedings No. 127, 1985, p. 243; L. R. Evans, Proceedings of the 12th International Conference on High-Energy Accelerators (Fermilab, 1983), p. 229; L. Bvans, Physics of Particle Accelerators (SLAC Summer School 1985 and Fermilab Summer School 1984), AIP Conference Proceedings No. 153,1987 , p. 1723.

[W] Mel Month, Collider Performance with Ideal Collisions, June 1, 1985 (Accel. Div. Report 85-1; DO Note 201).

[MOR] Vacuum and Beam Lifetime in the Doubler, 8. Mizuno et al., Fermilab Interaal Report UPC \#119 (1979). 